\title{
Central Nervous System Based Computing Models for Shelf Life Prediction of Soft Mouth Melting Milk Cakes
}

\author{
Sumit Goyal \\ Sr. Research Fellow, National Dairy Research Institute, Karnal, India \\ Email: thesumitgoyal@gmail.com \\ Gyanendra Kumar Goyal \\ Emeritus Scientist, National Dairy Research Institute, Karnal, India \\ Email:gkg5878@yahoo.com
}

\begin{abstract}
This paper presents the latency and potential of central nervous system based system intelligent computer engineering system for detecting shelf life of soft mouth melting milk cakes stored at $10^{\circ} \mathrm{C}$. Soft mouth melting milk cakes are exquisite sweetmeat cuisine made out of heat and acid thickened solidified sweetened milk. In today's highly competitive market consumers look for good quality food products. Shelf life is a good and accurate indicator to the food quality and safety. To achieve good quality of food products, detection of shelf life is important. Central nervous system based intelligent computing model was developed which detected $\mathbf{1 9 . 8 2}$ days shelf life, as against 21 days experimental shelf life.
\end{abstract}

Index Terms - central Nervous System, ANN, shelf life, soft mouth melting milk cakes, feedforward backpropagation, radial basis, artificial intelligence

\section{Introduction}

This paper presents the potential of central nervous system (CNS) based intelligent computer engineering methods of backpropagation and linear layer(design) for detecting shelf life of soft mouth melting milk cakes stored at $10^{\circ} \mathrm{C}$. Soft mouth melting milk cakes are exquisite sweetmeat cuisine made out of heat and acid thickened solidified sweetened milk. Soft mouth melting milk cakes are prepared from water buffalo milk standardized to $6 \%$ fat. The sweetmeat is manufactured in a double jacketed stainless steel kettle, and stored at $10^{\circ} \mathrm{C}$. The data samples for soft mouth melting milk cakes used in this study, relate to overall acceptability score obtained by evaluation of the product at regular intervals by a panel of well trained judges, and changes in physicochemical characteristics, viz., moisture, titratable acidity, free fatty acids, tyrosine content and peroxide value. An artificial neural network (ANN), usually called neural network (NN) is a mathematical model or computational model that is inspired by the structure and functional aspects of CNS. CNS based computing method is an adaptive system that changes its structure based on external or internal information that flows through the network during the learning phase.

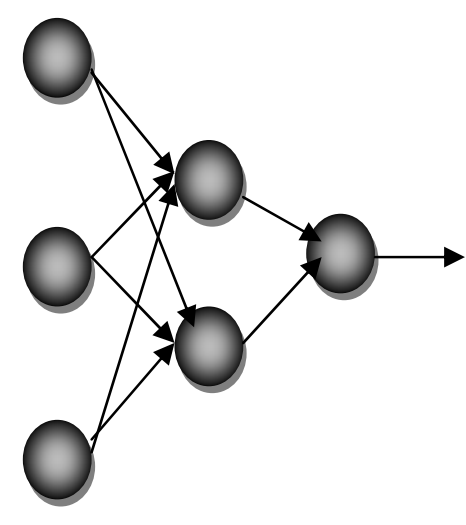

Figure 1. A structure of artificial neural network based on central nervous system

In CNS based intelligent computing, simple artificial nodes, variously called "neurons", "neurodes", "processing elements" or "units", are connected together to form a network of nodes mimicking the biological neural networks - hence the term "ANN" (Figure1). Generally, CNS involves a network of simple processing elements that exhibit complex global behavior determined by connections between processing elements and element parameters. While an ANN does not have to be adaptive, its practical use comes with algorithms designed to alter the weights of the connections in the network to produce a desired signal flow. ${ }^{[1]}$.

\subsection{Feedforward Backpropagation Method (FBM)}

Feedforward method allows signals to travel one way only, from input to output. There is no feedback (loops), i.e., the output of any layer does not affect that same layer. Feedforward CNSs tend to be straight forward networks that associate inputs with outputs. ${ }^{[2]}$.

\subsection{Linear Layer (Design) Method (LLDM)}

Linear layers are single layers of linear neurons. They may be static, with input delays of 0 , or dynamic, 
with input delays greater than 0 . They can be trained on simple linear time series problems, but often are used adaptively to continue learning while deployed, so they can adjust to changes in the relationship between inputs and outputs while being used ${ }^{[3]}$.

\subsection{Network layers}

The commonest type of ANN consists of three groups, or layers, of units: a layer of "input" units is connected to a layer of "hidden" units, which is connected to a layer of "output" units. The activity of the input units represents the raw information that is fed into the network. The behaviour of the output units depends on the activity of the hidden units and the weights between the hidden and output units. The weights between the input and hidden units determine when each hidden unit is active, and so by modifying these weights, a hidden unit can choose what it represents ${ }^{[2]}$.

\subsubsection{Single-layer and multi-layer architectures}

The single-layer organization, in which all units are connected to one another, constitutes the most general case and is of more potential computational power than hierarchically structured multi-layer organizations as represented in Figure 2.

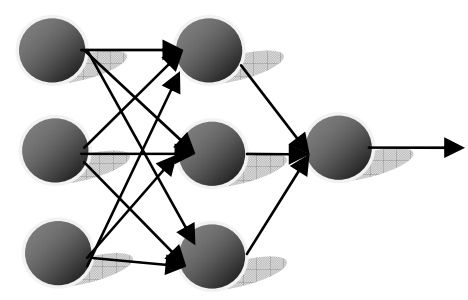

Figure 2. Single hidden layer architecture

In multi-layer networks, units are often numbered by layer, instead of following a global numbering supervised learning which incorporates an external teacher; so that each output unit is told what its desired response to input signals ought to be. Architecture of multiple hidden layer is displayed in Figure 3.

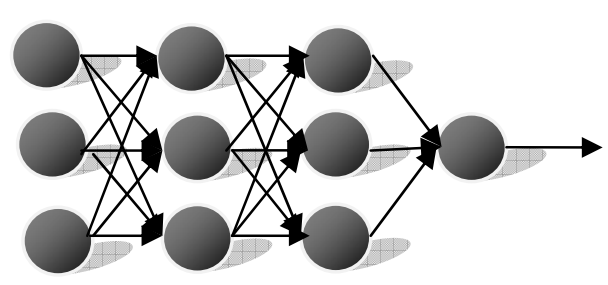

Figure 3. Two hidden layer architecture

\subsection{Importance of Shelf Life}

Shelf life of food products is an important factor for consumer's health. It is the recommendation of time that products can be stored, during which the defined quality of a specified proportion of the food remains acceptable under specified conditions of distribution, storage and display. Most shelf life labels or listed expiry dates are used as guidelines based on normal handling of products.

The use of product is dangerous and ineffective after the expiration date, as eating of such food may lead to food poisoning ${ }^{[4]}$. Detecting shelf life of a product in laboratory is quiet time consuming and expensive. Hence, it is important and relevant to develop such a system that detects shelf life of food products at low cost and in less time. Goyal and Goyal ${ }^{[5]}$ implemented artificial neural engineering and regressions models for forecasting shelf life of instant coffee drink, and compared both the models were with each other. From the investigation they concluded that multiple linear regression model was superior over radial basis model for forecasting shelf life of instant coffee drink. The predicted shelf life was found to be 37.29 days, i.e., within the experimentally obtained shelf life of 45 days. Goyal and Goyal ${ }^{[6]}$ developed linear layer (design) and time - delay methods of intelligent computing expert system for shelf life prediction of soft mouth melting milk cakes stored at $6^{\circ} \mathrm{C}$. They compared performances of the developed intelligent computing expert system models with each other, and observed that linear layer (design) model exhibited better results. The models predicted the shelf life as 49.54 days, which was very close to the experimentally obtained shelf life of 50 days.

Generalized regression and linear layer (Train) models predicted shelf life of milky white dessert jeweled with pistachio ${ }^{[7]}$. The predicted value was 20.15 days, i.e., within the experimentally obtained shelf life of 21 days, suggesting that ANN based linear layer train models are very effective for estimating the shelf life of milky white desserts jeweled with pistachio. Neuron based artificial intelligent scientific computer engineering models for estimating shelf life of instant coffee sterilized drink were developed by Goyal and Goyal ${ }^{[8]}$. Elman and Self-Organizing simulated neural network models predicted shelf life of soft cakes 20.57 days, whereas experimental shelf life was 21 days. Goyal and Goyal concluded that simulated neural networks can be used to predict shelf life of soft cakes stored at $10^{\circ} \mathrm{C}$ [9]. Computational ANN techniques for shelf life prediction of processed cheese have been developed ${ }^{[10]}$. In this paper we are presenting CNS based intelligent computer engineering system for detecting shelf life of soft mouth melting milk cake that would be quite useful to consumers, food industry, retailers, and the researchers.

\section{Method and material 2.1 Processing of Data}

The dataset was randomly divided into two disjoint subsets, namely, training set containing $80 \%$ of total observations and test set consisting of $20 \%$ of total observations. 


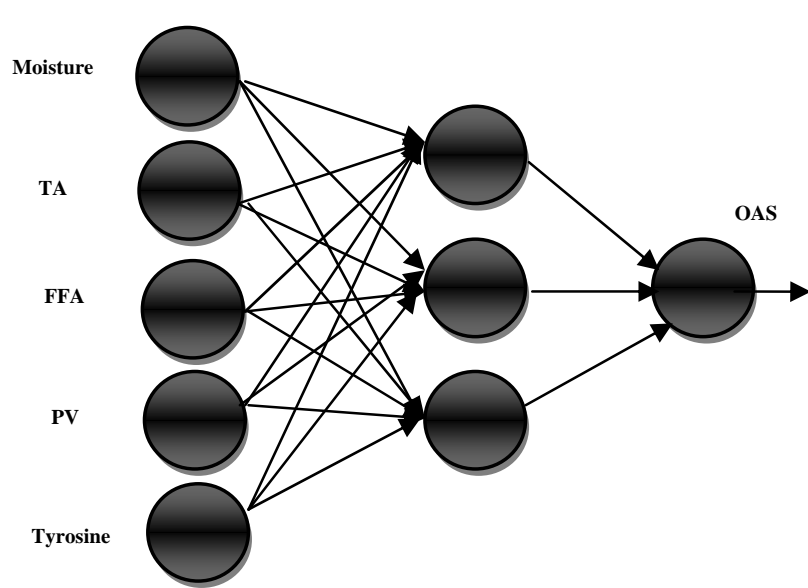

Figure 4. Input and output parameters for CNS models

The input parameters were Moisture, Titratable Acidity (TA), Free Fatty Acids (FFA), Peroxide Value (PV), and Tyrosine. The Overall Acceptability Score (OAS) was used as output parameter for developing the CNS intelligent systems (Figure 4).Training pattern for CNS intelligent systems is represented in Figure 5.

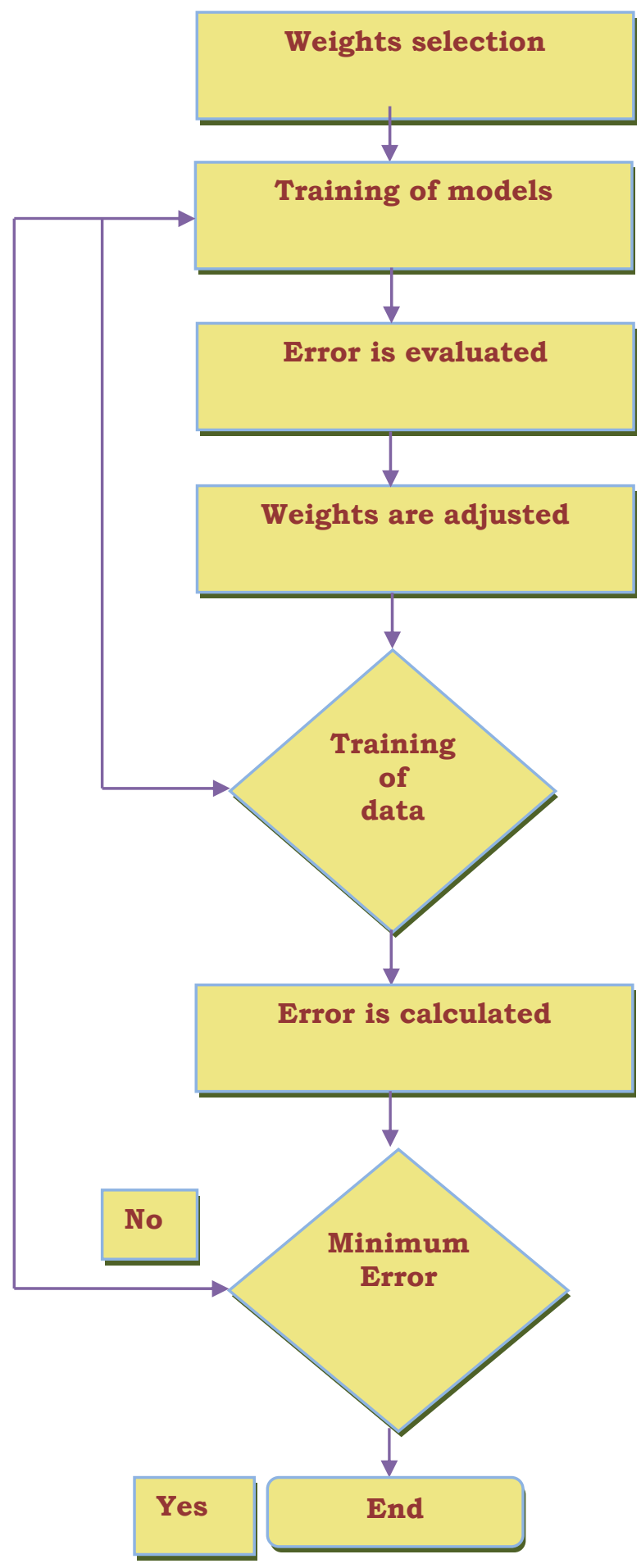

Figure 5. Training Pattern of the CNS's intelligent computing methods

\subsection{Training and Validation}

CNS intelligent engineering systems were developed by training the network with $80 \%$ of total observations and by validating with $20 \%$ of total observations. Different algorithms like Polak Ribiére 
Update conjugate gradient algorithm, Fletcher Reeves update conjugate gradient algorithm, Levenberg Marquardt algorithm, Gradient Descent algorithm with adaptive learning rate, Bayesian regularization, Powell Beale restarts conjugate gradient algorithm and BFG quasi-Newton algorithm were tried. Bayesian regularization algorithm gave good results; hence it was used as training function. Number of neurons in each hidden layer varied from 1 to 30. The network was trained with 1000 epochs. The system was trained with single as well as double hidden layers. Several problems were faced while training CNS intelligent engineering systems, too many neurons in the hidden layers resulted in overfitting. Overfitting occurs when the neural network has so much information processing capacity that the limited amount of information contained in the training set is not enough to train all of the neurons in the hidden layers. Using too few neurons in the hidden layers also resulted in underfitting. Underfitting occurs when there are too few neurons in the hidden layers to adequately detect the signals in a complicated dataset. A second problem can occur even when there is sufficient training data resulting in increase in training time of the network. Obviously some compromise must be reached between too many and too few neurons in the hidden layers. Ultimately the selection of the architecture of neural network came down to trial and error. There are two trial and error approaches that are used in determining the numbers of hidden neurons: the "forward" and "backward" selection methods. The first method, the "forward selection method", begins by selecting a small number of hidden neurons. The second method, the "backward selection method", begins by using a large number of hidden neurons. Then the neural network is trained and tested. This process continues until about the performance improvement of the neural network is no longer significant ${ }^{[11]}$.

\section{Results and Discussion}

Table 1: Results of FBM with single hidden layer

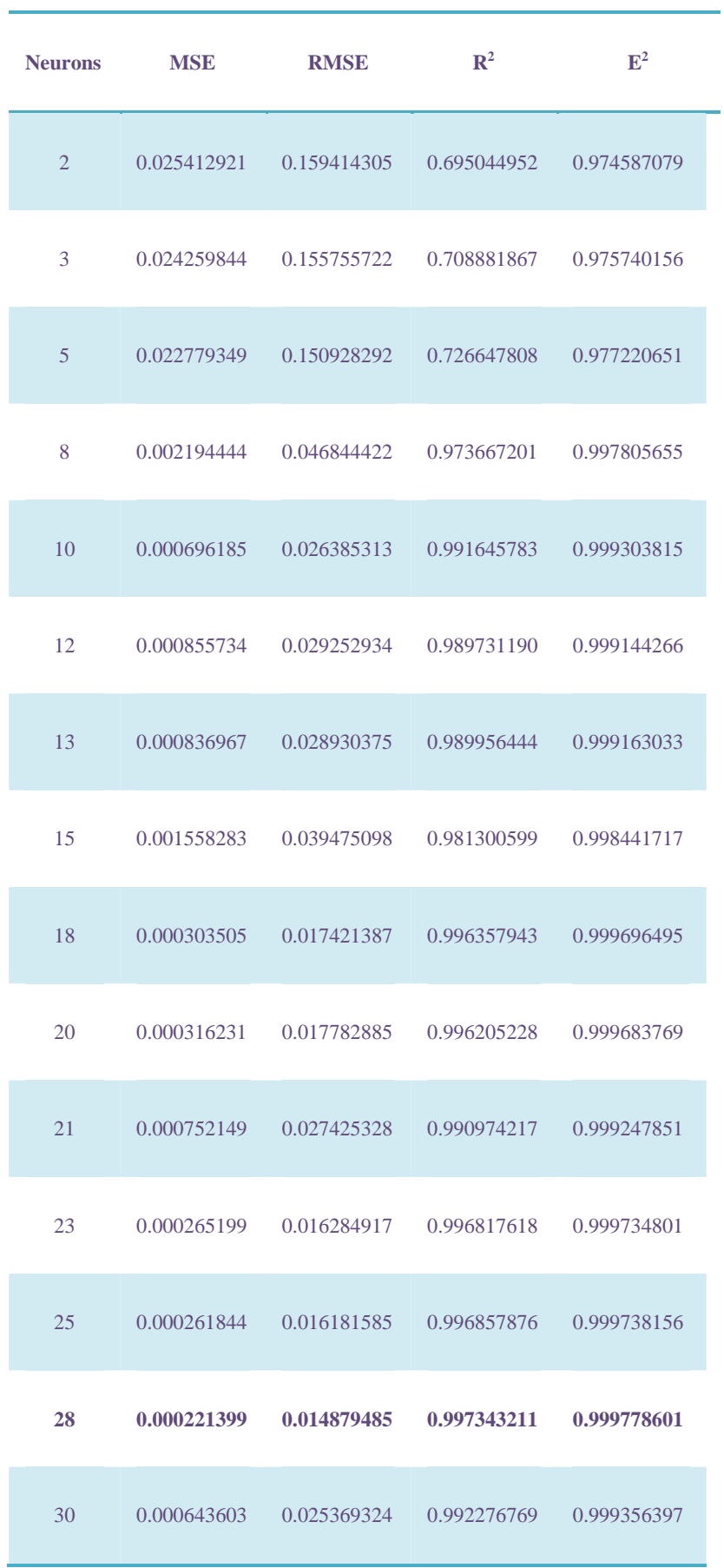


Table 2 : Results of FBM with two hidden layers

\begin{tabular}{|c|c|c|c|c|}
\hline Neurons & MSE & RMSE & $\mathbf{R}^{2}$ & $\mathbf{E}^{2}$ \\
\hline $3: 3$ & 0.001615840 & 0.040197515 & 0.980609918 & 0.998384160 \\
\hline $5: 5$ & 0.005742624 & 0.075780104 & 0.931088510 & 0.994257376 \\
\hline $7: 7$ & 0.001328440 & 0.036447777 & 0.984058714 & 0.998671560 \\
\hline $8: 8$ & 0.002305860 & 0.048019371 & 0.972329688 & 0.997694140 \\
\hline $10: 10$ & 0.001551838 & 0.039393379 & 0.981377940 & 0.998448162 \\
\hline $12: 12$ & 0.002430342 & 0.049298555 & 0.970835895 & 0.997569658 \\
\hline $14: 14$ & 0.000425775 & 0.020634323 & 0.994890698 & 0.999574225 \\
\hline $15: 15$ & 0.001045304 & 0.032331169 & 0.987456346 & 0.998954696 \\
\hline $18: 18$ & 0.000446558 & 0.021131914 & 0.994641307 & 0.999553442 \\
\hline $20: 20$ & 0.000498923 & 0.022336592 & 0.994012922 & 0.999501077 \\
\hline
\end{tabular}

Table 3 : Results of LLDM

\begin{tabular}{cccc}
\hline MSE & RMSE & $\mathrm{R}^{2}$ & $\mathrm{E}^{2}$ \\
\hline & & & \\
\hline 0.000715736 & 0.026753243 & 0.991411168 & 0.999284264 \\
\hline
\end{tabular}

layer, FBM system having fourteen neurons in the first layer and second layer gave the best results (MSE: 0.000425775, RMSE : $\mathbf{0 . 0 2 0 6 3 4 3 2 3 ,} \mathrm{R}^{2}: \mathbf{0 . 9 9 4 8 9 0 6 9 8}$, $\mathrm{E}^{2}$ : 0.999574225). LLDM system was also developed and its best performance was MSE: 0.000715736, RMSE: 0.026753243, $\mathrm{R}^{2}$ : $\mathbf{0 . 9 9 1 4 1 1 1 6 8 ,} \mathrm{E}^{2}$ : 0.999284264. Actual Overall Acceptability Score (AOAS) and Predicted Overall Acceptability Score (POAS) for single layer and multilayer FBM models are illustrated in Figure 6 and Figure7, Figure 8 represents AOAS and POAS for LLDM model.

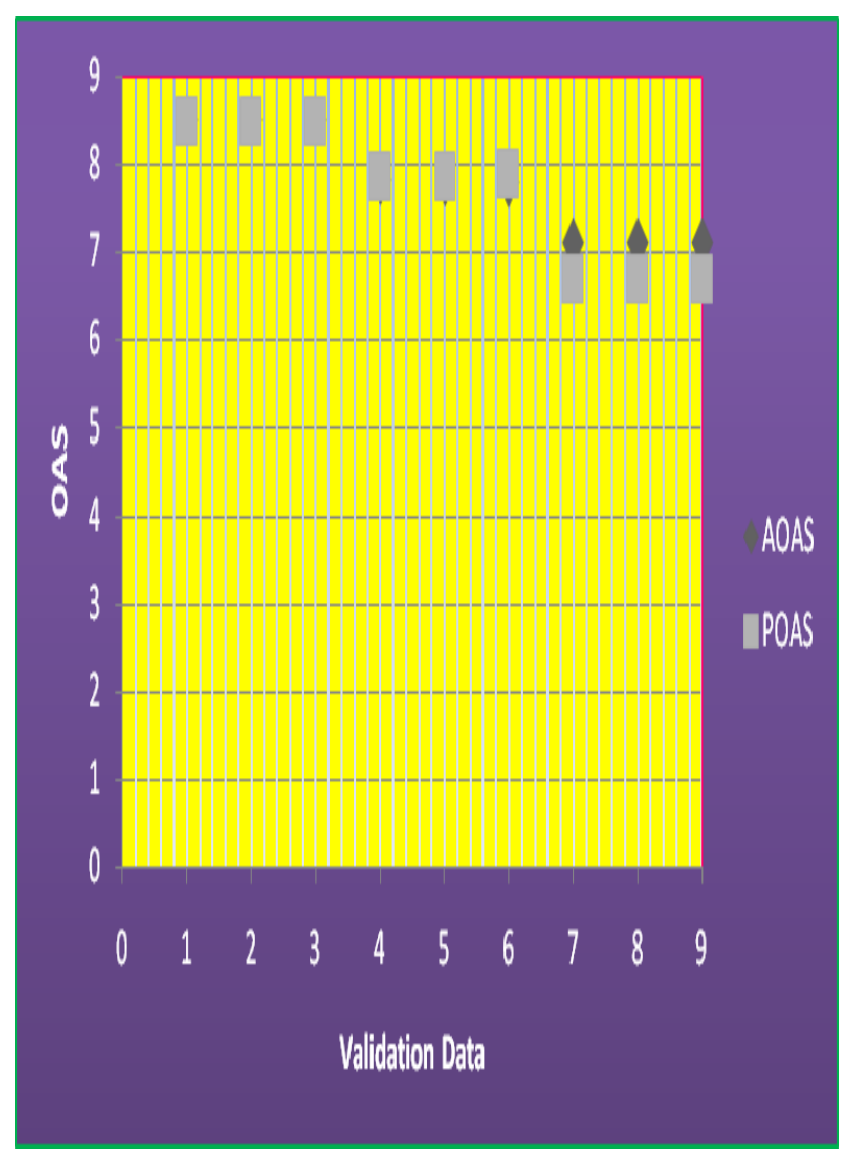

Figure 6. Comparison of AOAS and POAS for single layer FBM model
CNS based FBM and LLDM systems were developed for detecting shelf life of soft mouth melting milk cakes as shown in Table 1,2 and 3 respectively. Numerous experiments were carried out with single as well as double hidden layers for FBM system. Several different topologies were tried and tested. For testing efficiency of CNS intelligent computer engineering models, four different prediction performance measures, viz. MSE, RMSE, $\mathrm{R}^{2}$ and $\mathrm{E}^{2}$ were used. It was observed that FBM system with single hidden layer having twenty eight neurons gave the best results ( MSE : 0.000221399, RMSE : 0.014879485, $\mathrm{R}^{2}$ : 0.997343211, $\mathrm{E}^{2}: \mathbf{0 . 9 9 9 7 7 8 6 0 1}$ ) and for double hidden 


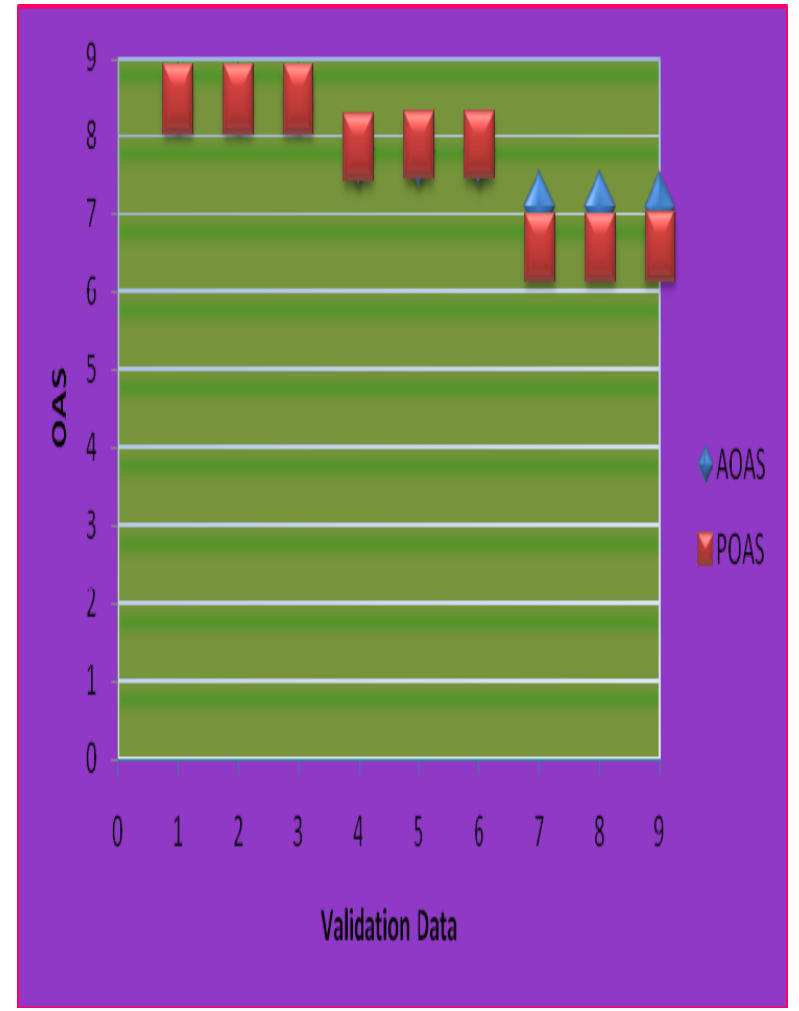

Figure 7. Comparison of AOAS and POAS for multi layer FBM model

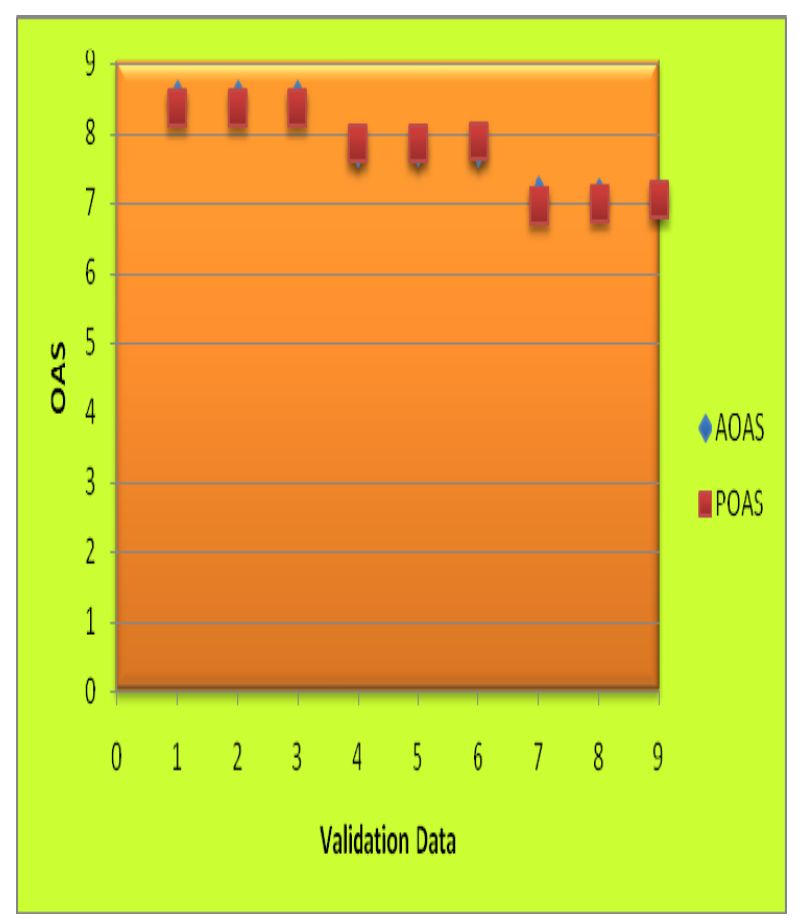

Figure 8. Comparison of AOAS and POAS for multi layer LLDM model

Regression equations were developed to estimate shelf life of the soft mouth melting milk cakes, i.e., in days for which product has been in the shelf based on overall acceptability score, taking storage intervals (in days) as dependent variable and overall acceptability score as independent variable. $R^{2}$ was found to be 96.1 percent of the total variation as explained by overall acceptability scores (Figure 9).

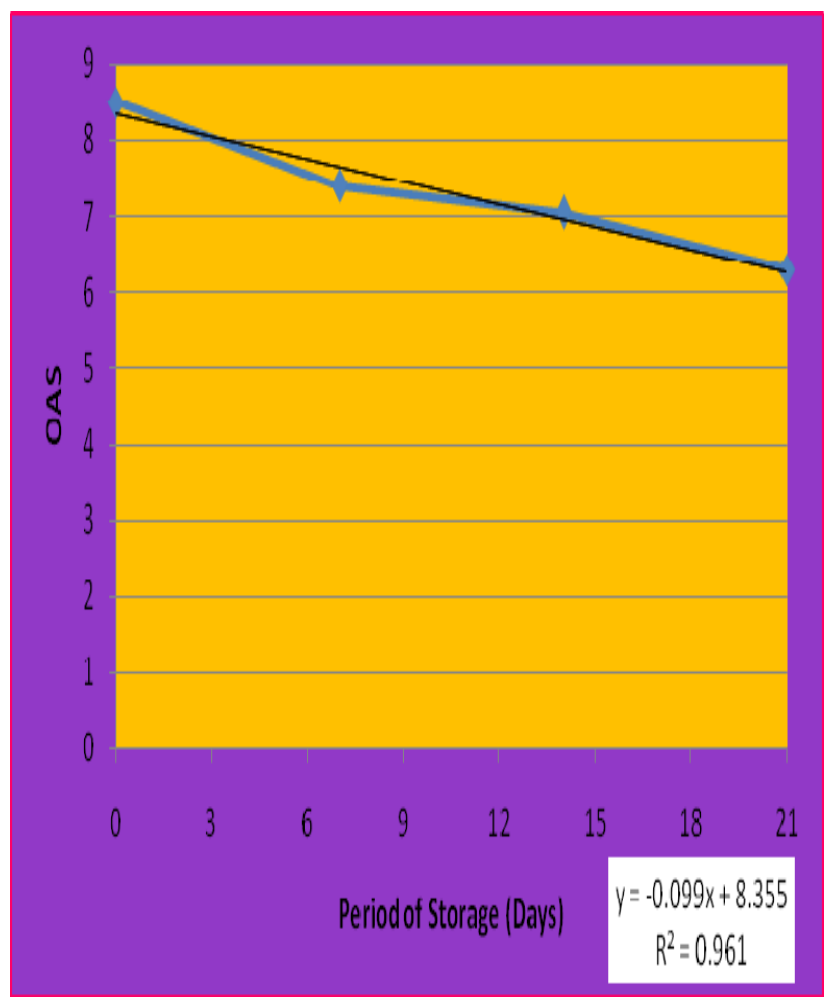

Figure 9. Shelf life predicted by regression equations

The shelf life is computed by subtracting the obtained value of days from experimentally determined shelf life, which was found to be 19.82 days. The predicted value is very close to the experimentally obtained shelf life of 21 days. From the study, it is evident that this new field of CNS based intelligent computer engineering is quite efficient in detecting the shelf life of soft mouth melting milk cakes.

\section{Conclusion}

In today's highly competitive market shelf life of product is a good and accurate indicator of the food quality and safety. For achieving good quality and safety of food products, detection of shelf life is important. In this paper CNS based intelligent computer engineering system has been developed to detect shelf life of soft mouth melting milk cakes stored at $10^{\circ} \mathrm{C}$. Feedforward backpropagation single layer and multilayer models along with Linear Layer (Design) models were developed and compared with each other. The dataset was randomly divided into two disjoint subsets, namely, training set containing $80 \%$ of the total observations and testing set consisting of $20 \%$ of total observations. The input parameters were Moisture, Titratable Acidity, Free Fatty Acids, Peroxide Value, and Tyrosine. The Overall Acceptability Score was taken as output parameter for developing the CNS intelligent systems. Number of 
neurons in each hidden layer varied from 1 to 30. The network was trained with 1000 epochs. Mean Square Error, Root Mean Square Error, Coefficient of Determination and Nash - Sutcliffo Coefficient were used in order to compare the prediction ability of the developed models. It was observed that feedforward model with single hidden layer having twenty eight neurons gave the best result. Based on these results regression equations were developed for predicting shelf life, which came out as 19.82 days vis-à-vis 21 days experimental shelf life. Hence, from the study it is concluded that central nervous system based intelligent computer engineering system is quite effective in forecasting the shelf life of soft mouth melting milk cakes.

\section{References}

[1] http://en.wikipedia.org/wiki/Artificial_neural_network (accessed on 28.8.2011)

[2]http://www.doc.ic.ac.uk/ nd/surprise_96/journal/vol4/cs11/r eport.html(accessed on 29.8.2011)

[3] http://www.mathworks.com/help/toolbox/nnet/ref/ linearlayer.html. (accessed on 18.8.2011)

[4] http://en.wikipedia.org/wiki/Shelf_life (accessed on 1.12.2011)

[5] Goyal Sumit and Goyal G K. Application of artificial neural engineering and regression models for forecasting shelf life of instant coffee drink [J]. International Journal of Computer Science Issues, 2011, 8(4), 1, 320-324

[6] Goyal Sumit and Goyal G K. Development of intelligent computing expert system models for shelf life prediction of soft mouth melting milk cakes [J].International Journal of Computer Applications 2011, 25(9), 41-44

[7] Goyal Sumit and Goyal G K. A new scientific approach of intelligent artificial neural network engineering for predicting shelf life of milky white dessert jeweled with pistachio [J]. International Journal of Scientific and Engineering Research, 2011, 2(9), 1-4

[8] Goyal Sumit and Goyal G K. Development of neuron based artificial intelligent scientific computer engineering models for estimating shelf life of instant coffee sterilized drink [J]. International Journal of Computational Intelligence and Information Security, 2011, 2(7), 4 - 12

[9] Goyal Sumit and Goyal G K. Simulated neural network intelligent computing models for predicting shelf life of soft cakes [J]. Global Journal of Computer Science and Technology, 2011, 11(14), Version 1.0, 29-33

[10] Goyal Sumit and Goyal G K. Performance of generalized regression, radial basis (fewer neurons), and linear layer (design) computational ANN techniques for shelf life prediction of processed cheese [J]. International Journal of Artificial Intelligence and Knowledge Discovery, 2011, 1(4), 12-15.

[11]http://www.heatonresearch.com/articles/5/page2.html. (accessed on 30.8.2011)

\section{Author's Biodata}

Sumit Goyal: is Senior Research Fellow at National Dairy Research Institute, Karnal (Haryana) India. His research interests have been in the area of artificial neural networks and prediction of shelf life of food products. His research has appeared in Canadian Journal on Artificial Intelligence, Machine Learning and Pattern Recognition, International Journal of Computer Applications, International Journal of Computational Intelligence and Information Security, International Journal of Latest Trends in Computing, International Journal of Scientific and Engineering Research, International Journal of Computer Science Issues, International Journal of Computer Science \& Emerging Technologies, Global Journal of Computer Science and Technology, International Journal of Artificial Intelligence and Knowledge Discovery, amongst others. He is member of IDA.

Gyanendra Kumar Goyal: is Emeritus Scientist at National Dairy Research Institute, Karnal, India. His research interests include dairy \& food packaging and shelf life determination of food products. His research work has been published in Int. J. of Food Sci. Technol. and Nutrition, Nutrition and Food Science, Milchwissenschaft, American Journal of Food Technology, British Food Journal, Canadian Journal on Artificial Intelligence, Machine Learning and Pattern Recognition, International Journal of Computer Applications, International Journal of Computational Intelligence and Information Security, International Journal of Latest Trends in Computing, International Journal of Scientific and Engineering Research, International Journal of Computer Science Issues, International Journal of Computer Science \& Emerging Technologies, Global Journal of Computer Science and Technology, International Journal of Artificial Intelligence and Knowledge Discovery amongst others. He is life member of AFST (I) and IDA. 